\title{
Properties of the stochastic Gross-Pitaevskii equation: finite temperature Ehrenfest relations and the optimal plane wave representation
}

\author{
A S Bradley ${ }^{1,3}$, P B Blakie ${ }^{2}$ and C W Gardiner ${ }^{1,2}$ \\ ${ }^{1}$ School of Chemical and Physical Sciences, Victoria University of Wellington, New Zealand \\ 2 Ultra-Cold Atoms Group, Department of Physics, University of Otago, Dunedin, New Zealand \\ ${ }^{3}$ ARC Center of Excellence for Quantum-Atom Optics, University of Queensland, Brisbane, \\ QLD 4072, Australia \\ E-mail: abradley@physics.uq.edu.au
}

Received 16 September 2005, in final form 18 October 2005

Published 14 November 2005

Online at stacks.iop.org/JPhysB/38/4259

\begin{abstract}
We present Ehrenfest relations for the high temperature stochastic GrossPitaevskii equation description of a trapped Bose gas, including the effect of growth noise and the energy cutoff. A condition for neglecting the cutoff terms in the Ehrenfest relations is found which is more stringent than the usual validity condition of the truncated Wigner or classical field method-that all modes are highly occupied. The condition requires a small overlap of the nonlinear interaction term with the lowest energy single particle state of the noncondensate band, and gives a means to constrain dynamical artefacts arising from the energy cutoff in numerical simulations. We apply the formalism to two simple test problems: (i) simulation of the Kohn mode oscillation for a trapped Bose gas at zero temperature, and (ii) computing the equilibrium properties of a finite temperature Bose gas within the classical field method. The examples indicate ways to control the effects of the cutoff, and that there is an optimal choice of plane wave basis for a given cutoff energy. This basis gives the best reproduction of the single particle spectrum, the condensate fraction and the position and momentum densities.
\end{abstract}

\section{Introduction}

The description of weakly interacting Bose gases using some form of the nonlinear Schrödinger equation has a long history, beginning with the zero temperature treatment now known as the Gross-Pitaevskii equation (GPE) [1-3]. A number of studies have used the GPE to investigate the dynamics of Bose-Einstein condensation (BEC) [4-7], leading to the prediction that the condensation process is associated with waves in energy space which propagate from high to low energy [8, 9]. A quantum kinetic theory (QKT) of BEC was developed in a series of papers by Gardiner et al [10-20], which has some aspects of its formulation in common with 
the kinetic description of BEC arising from the GPE in the random phase approximation [21]. Indeed, the scaling behaviour of the energy distribution predicted by QKT [15] is in good agreement with the theory of $[8,9]$.

While these theories have been quite successful in many respects, in particular, the accurate description of Bose-Einstein condensation in certain regimes [19, 20], there are physical aspects of Bose-Einstein condensates which require alternative approaches, most notably the combined effect of nonlinear interactions, finite temperatures and quantum coherences. Exact methods based on the positive-P representation [22-25], in principle describe all of the quantum coherences and finite temperature many-body physics of the process, but can still present significant technical challenges. A popular approach, which leads to a computationally tractable, but nevertheless approximate description, entails the assumption that certain modes of a partially condensed Bose gas are highly occupied - a condition which is very often satisfied during Bose-Einstein condensation. We refer to such approaches as classical field methods [21, 23, 26-43] which have a similar relationship to the theory of dilute Bose gases as the classical field theory of the optical laser has to laser theory [44].

In this paper, we investigate a subset of these approaches, which we will call the stochastic Gross-Pitaevskii equation (SGPE) [28, 29, 38, 39], and as special cases we will also discuss the projected Gross-Pitaevskii equation (PGPE) method [21,35-37] and the damped GPE $[38,45,46]$. Our main aim is to derive the Ehrenfest relations for the SGPE describing a condensate in collisional contact with a high temperature thermal cloud. As a necessary part of the formalism we include the effect of thermal fluctuations, and the energy cutoff used to separate the atoms treated as an incoherent thermal gas from those that make up the highly occupied classical field region. A central feature of this work is the investigation of the consequences of using different representations of the classical field region, both on the evolution of operator averages and on the equilibrium properties of the gas.

This paper is structured as follows. The GPE and PGPE are introduced in section 2, and the Ehrenfest relations for the GPE are briefly reviewed. In section 3 we outline the derivation of the SGPE with particular emphasis on the approximations involved. In section 4 we derive the Ehrenfest relations for the SGPE, and the GPE, PGPE and Gardiner-AnglinFudge equation [39] are discussed as special cases. As an application of the formalism, in section 5 we examine the effect of the projector terms on the Kohn mode oscillations of a trapped BEC modelled using the PGPE classical field method, and show how the artefacts may be controlled using our formalism. In section 6 we compute some properties of a finite temperature trapped Bose gas, investigating the effect of using different representations; a simple construction is shown to give the optimal plane wave representation. We conclude in section 7.

\section{Background}

It is well known that a solution of the GPE obeys the same Ehrenfest relations which hold for the Schrödinger equation [47]. We briefly reiterate these here to establish notation, and introduce the projected Gross-Pitaevskii equation.

\subsection{The Gross-Pitaevskii equation}

The Gross-Pitaevskii equation is the equation of motion for a complex scalar field evolving according to the Gross-Pitaevskii Hamiltonian functional

$$
H_{\mathrm{GP}}=\int \mathrm{d}^{3} \mathbf{x} \alpha^{*}(\mathbf{x}, t)\left(-\frac{\hbar^{2} \nabla^{2}}{2 m}+V(\mathbf{x}, t)+\frac{u}{2}|\alpha(\mathbf{x}, t)|^{2}\right) \alpha(\mathbf{x}, t),
$$


obtained by functional differentiation,

$$
\mathrm{i} \hbar \frac{\partial \alpha(\mathbf{x}, t)}{\partial t}=\frac{\delta H_{\mathrm{GP}}}{\delta \alpha^{*}(\mathbf{x}, t)}=L_{\mathrm{GP}} \alpha(\mathbf{x}, t),
$$

where we have defined the nonlinear Gross-Pitaevskii operator as

$$
L_{\mathrm{GP}} \alpha(\mathbf{x}) \equiv\left(-\frac{\hbar^{2} \nabla^{2}}{2 m}+V(\mathbf{x}, t)+u|\alpha(\mathbf{x})|^{2}\right) \alpha(\mathbf{x}) .
$$

In this paper $V(\mathbf{x}, t)$ describes the time-dependent external trapping potential, and $u \equiv 4 \pi \hbar^{2} a / m$ determines the two-body interaction strength in the cold collision regime [48], where $a$ is the $S$-wave scattering length and $m$ is the atomic mass. In addition to $H_{\mathrm{GP}}$, the other quantities of interest are position, momentum and angular momentum $(\hat{\mathbf{x}}, \hat{\mathbf{p}}, \hat{\mathbf{L}})$ which have the usual Schrödiner forms, and the occupation number $N_{\mathrm{GP}}$ :

$$
N_{\mathrm{GP}}=\int \mathrm{d}^{3} \mathbf{x} \alpha^{*}(\mathbf{x}, t) \alpha(\mathbf{x}, t) .
$$

For brevity, we will denote the spatial expectation value of an operator $A$ with respect to the condensate wavefunction by

$$
\overline{\hat{A}} \equiv \int \mathrm{d}^{3} \mathbf{x} \alpha^{*}(\mathbf{x}, t) \hat{A} \alpha(\mathbf{x}, t),
$$

and we will drop the 'hat' notation, since it will always be clear from the context when we are dealing with operators. In this notation, the Ehrenfest equations for the Gross-Pitaevskii equation are

$$
\begin{aligned}
& \frac{\mathrm{d} \overline{\mathbf{x}}}{\mathrm{d} t}=\frac{\overline{\mathbf{p}}}{m} \\
& \frac{\mathrm{d} \overline{\mathbf{p}}}{\mathrm{d} t}=-\overline{\nabla V(\mathbf{x}, t)} \\
& \frac{\mathrm{d} \overline{\mathbf{L}}}{\mathrm{d} t}=-\frac{\mathrm{i}}{\hbar} \overline{\mathbf{L} V(\mathbf{x}, t)} \\
& \frac{\mathrm{d} H_{\mathrm{GP}}}{\mathrm{d} t}=\frac{\partial V(\mathbf{x}, t)}{\partial t} \\
& \frac{\mathrm{d} N_{\mathrm{GP}}}{\mathrm{d} t}=0 .
\end{aligned}
$$

\subsection{The projected Gross-Pitaevskii equation}

The projector that separates the trapped states into upper and lower energy bands is defined by first separating the potential into

$$
V(\mathbf{x}, t) \equiv V_{0}(\mathbf{x})+\delta V(\mathbf{x}, t)
$$

where the time invariant potential $V_{0}(\mathbf{x})$ is used to define the single particle Hamiltonian operator

$$
H_{0} \equiv \frac{-\hbar^{2} \nabla^{2}}{2 m}+V_{0}(\mathbf{x})
$$

and the time-dependent potential $\delta V(\mathbf{x}, t)$ is arbitrary. The representation basis is provided by the energy eigenstates satisfying

$$
H_{0} Y_{n}(\mathbf{x})=\epsilon_{n} Y_{n}(\mathbf{x}),
$$


where the index $n$ represents the complete set of quantum numbers required to describe the basis in a particular choice of coordinates, and $\epsilon_{n}$ is the energy eigenvalue. We have avoided using the terminology single particle basis because in practice there are many possible choices of basis for the same single particle Hamiltonian.

2.2.1. Projectors. The operator which projects into the condensate band is defined by its action on an arbitrary function of position $\chi(\mathbf{x})$, as

$$
\begin{aligned}
\mathcal{P} \chi(\mathbf{x}) & \equiv \overline{\sum_{n}} Y_{n}(\mathbf{x}) \int \mathrm{d}^{3} \mathbf{z} Y_{n}^{*}(\mathbf{z}) \chi(\mathbf{z}) \\
& =\int \mathrm{d}^{3} \mathbf{z} \delta_{C}(\mathbf{x}, \mathbf{z}) \chi(\mathbf{z}) .
\end{aligned}
$$

In defining $\mathcal{P}$ we have made use of the projected delta-function

$$
\delta_{C}(\mathbf{x}, \mathbf{z}) \equiv \overline{\sum_{n}} Y_{n}^{*}(\mathbf{z}) Y_{n}(\mathbf{x}),
$$

and the notation

$$
\overline{\sum_{n}} \equiv \sum_{\epsilon_{n} \leqslant E_{R}}
$$

represents the sum over all single particle states with energy less than the cutoff. In the summation $E_{R}$ is the cutoff energy. The projector orthogonal to $\mathcal{P}$ is

$$
\mathcal{Q} \chi(\mathbf{x}) \equiv(1-\mathcal{P}) \chi(\mathbf{x})=\sum_{\epsilon_{n}>E_{R}} Y_{n}(\mathbf{x}) \int \mathrm{d}^{3} \mathbf{z} Y_{n}^{*}(\mathbf{z}) \chi(\mathbf{z}) .
$$

We will also find it convenient to define the complex conjugate projector

$$
\mathcal{Q}^{*} \chi(\mathbf{y}) \equiv \sum_{\epsilon_{n}>E_{R}} Y_{n}^{*}(\mathbf{x}) \int \mathrm{d}^{3} \mathbf{z} Y_{n}(\mathbf{z}) \chi(\mathbf{z}) .
$$

The projectors satisfy the identities

$$
\begin{aligned}
& \mathcal{Q P}=0, \\
& \mathcal{Q} H_{0} \mathcal{P}=0, \\
& \int \mathrm{d}^{3} \mathbf{x} \phi^{*} \mathcal{Q} \chi=\int \mathrm{d}^{3} \mathbf{x}(\mathcal{Q} \phi)^{*} \chi,
\end{aligned}
$$

where the first is just orthogonality, the second is a consequence of orthogonality and the composition of the projector in terms of eigenstates of $H_{0}$, and the third expresses the Hermitian nature of the projectors.

2.2.2. The PGPE. We decompose the classical field in terms of the mode amplitudes $\alpha_{n}(t)$

$$
\alpha(\mathbf{x}, t) \equiv \overline{\sum_{n}} \alpha_{n}(t) Y_{n}(\mathbf{x}),
$$

where the field is now projected into the region below the cutoff. If we reinterpret (1) with the replacement $\alpha \rightarrow \mathcal{P} \alpha$, the PGPE is derived by functional differentiation of (1) using the projected functional derivatives

$$
\frac{\bar{\delta}}{\bar{\delta} \alpha(\mathbf{x})} \equiv \overline{\sum_{n}} Y_{n}^{*}(\mathbf{x}) \frac{\partial}{\partial \alpha_{n}}, \quad \quad \frac{\bar{\delta}}{\bar{\delta} \alpha^{*}(\mathbf{x})} \equiv \overline{\sum_{n}} Y_{n}(\mathbf{x}) \frac{\partial}{\partial \alpha_{n}^{*}} .
$$




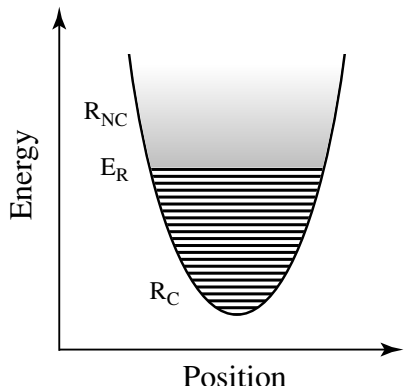

(a) Separation

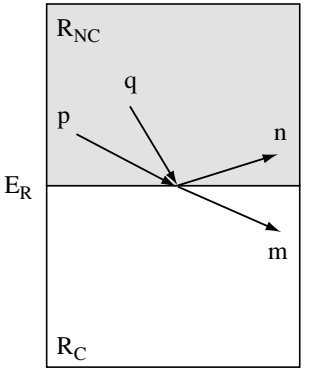

(b) Growth

Figure 1. (a) Schematic of the cutoff energy $E_{R}$ separating states according to their energy. States beneath $E_{R}$ form the condensate band which warrants a quantum mechanical description. States above $E_{R}$ form the noncondensate band which is treated as thermalized. (b) Schematic of the energy and momentum conserving collisions which cause particle exchange between bands. A collision between two noncondensate atoms imparts most of the collision energy to one atom which remains in the noncondensate band. The low energy atom passes into the condensate band where it contributes to condensate growth and thermal fluctuations. The time reversed loss process also occurs.

The PGPE then takes the form

$$
\mathrm{i} \hbar \frac{\partial \alpha(\mathbf{x}, t)}{\partial t}=\frac{\bar{\delta} H_{\mathrm{GP}}}{\bar{\delta} \alpha^{*}(\mathbf{x})}=\mathcal{P} L_{\mathrm{GP}} \alpha(\mathbf{x}, t)=(1-\mathcal{Q}) L_{\mathrm{GP}} \alpha(\mathbf{x}, t)
$$

Note that we have used $\mathcal{Q}$ to cast the PGPE in terms of the GPE plus an extra term arising from the projection. We can now find the Ehrenfest relations for the PGPE using (20)-(22) along with (25), and this is the basic course we follow in the next section. However, in order to describe fluctuations arising from the thermal cloud we will first introduce the SGPE.

\section{The stochastic Gross-Pitaevskii equation}

The SGPE formalism [39] separates the partially condensed system into a low energy subspace of modes (the condensate band), and its orthogonal complement (the noncondensate band), the union of which furnishes a complete basis. The noncondensate band is assumed thermalized, so that it may be described by Gaussian statistics and traced out. The noncondensate band thus plays the role of a thermal reservoir and acts as a damping mechanism for the condensate band, while the condensate band contains the condensate and its low energy excitations. The separation into bands is shown schematically in figure 1(a).

In this paper, we neglect the phase damping processes which lead to the scattering terms in the master equation of [39]. The physical process associated with these terms arises from scattering between an atom in the noncondensate band with one in the condensate band which leads to one of the atoms returning to each band. This process is not directly involved in condensate growth, and indeed it has been shown by Anglin and Zurek that the net effect of including these terms is a slight shift of the effective condensate growth rate [49]. While the full SGPE description is somewhat more complicated than the PGPE theory [21, 35-37], if we neglect the scattering term and also take the limit of a broad thermal cloud, it can be reduced to a relatively simple equation of motion for the condensate band which is closely related to the PGPE. The complete derivation of the SGPE may be found in [39]. Here we will briefly sketch the derivation, with a few minor changes of notation to make a transparent connection with the results to follow. 


\subsection{Hamiltonian}

To obtain the SGPE we proceed from the second quantized Hamiltonian for the system in the S-wave scattering limit

$H=\int \mathrm{d}^{3} \mathbf{x} \Psi^{\dagger}(\mathbf{x})\left(-\frac{\hbar^{2} \nabla^{2}}{2 m}+V(\mathbf{x}, t)\right) \Psi(\mathbf{x})+\frac{u}{2} \int \mathrm{d}^{3} \mathbf{x} \Psi^{\dagger}(\mathbf{x}) \Psi^{\dagger}(\mathbf{x}) \Psi(\mathbf{x}) \Psi(\mathbf{x})$.

The field operator is split at the cutoff energy into

$$
\Psi(\mathbf{x})=\phi(\mathbf{x})+\psi_{\mathrm{NC}}(\mathbf{x})=\mathcal{P} \Psi(\mathbf{x})+\mathcal{Q} \Psi(\mathbf{x}),
$$

where the noncondensate field operator $\psi_{\mathrm{NC}}(\mathbf{x})$ describes the high energy thermal modes. The commutator of the condensate band field operator is

$$
\left[\phi(\mathbf{x}), \phi^{\dagger}(\mathbf{y})\right]=\delta_{C}(\mathbf{x}, \mathbf{y})
$$

which is also the kernel of the projection operator defined in (16).

\subsection{Condensate band master equation}

The thermal statistics of the noncondensate field allow averages over many noncondensed field operators to be factorized and reduced to products of single particle Wigner functions $F(\mathbf{u}, \mathbf{v})$. The growth/loss master equation for the reduced density matrix of the condensate band $\rho_{C}=\operatorname{Tr}_{\mathrm{NC}}(\rho)$ can be written in terms of the amplitudes 4

$$
\begin{aligned}
G^{(+)}(\mathbf{u}, \mathbf{v}, \epsilon) & =\frac{u^{2}}{(2 \pi)^{8} \hbar^{2}} \int \mathrm{d}^{3} \mathbf{K}_{1} \int \mathrm{d}^{3} \mathbf{K}_{2} \int \mathrm{d}^{3} \mathbf{K}_{3} F\left(\mathbf{u}, \mathbf{K}_{1}\right) F\left(\mathbf{u}, \mathbf{K}_{2}\right)\left[1+F\left(\mathbf{u}, \mathbf{K}_{3}\right)\right] \\
& \times \delta\left(\omega_{1}+\omega_{2}-\omega_{3}-\epsilon / \hbar\right) \exp \left(-\mathrm{i}\left(\mathbf{K}_{1}+\mathbf{K}_{2}-\mathbf{K}_{3}\right) \cdot \mathbf{v}\right)
\end{aligned}
$$

and

$$
G^{(-)}(\mathbf{u}, \mathbf{v}, \epsilon)=\mathrm{e}^{(\epsilon-\mu) / k_{B} T} G^{(+)}(\mathbf{u}, \mathbf{v}, \epsilon)
$$

in the form ${ }^{5}$

$$
\begin{aligned}
\left.\dot{\rho}_{C}\right|_{\text {growth }}= & \int \mathrm{d}^{3} \mathbf{u} \int \mathrm{d}^{3} \mathbf{v}\left[\left\{G^{(-)}\left(\mathbf{u}, \mathbf{v}, L_{C}\right) \phi(\mathbf{u}-\mathbf{v} / 2)\right\} \rho_{C}, \phi^{\dagger}(\mathbf{u}+\mathbf{v} / 2)\right] \\
& -\int \mathrm{d}^{3} \mathbf{u} \int \mathrm{d}^{3} \mathbf{v}\left[\rho_{C}\left\{G^{(-)}\left(\mathbf{u}, \mathbf{v},-L_{C}\right) \phi^{\dagger}(\mathbf{u}-\mathbf{v} / 2)\right\}, \phi(\mathbf{u}+\mathbf{v} / 2)\right] \\
& +\int \mathrm{d}^{3} \mathbf{u} \int \mathrm{d}^{3} \mathbf{v}\left[\left\{G^{(+)}\left(\mathbf{u}, \mathbf{v},-L_{C}\right) \phi^{\dagger}(\mathbf{u}-\mathbf{v} / 2)\right\} \rho_{C}, \phi(\mathbf{u}+\mathbf{v} / 2)\right] \\
& -\int \mathrm{d}^{3} \mathbf{u} \int \mathrm{d}^{3} \mathbf{v}\left[\rho_{C}\left\{G^{(+)}\left(\mathbf{u}, \mathbf{v}, L_{C}\right) \phi(\mathbf{u}-\mathbf{v} / 2)\right\}, \phi^{\dagger}(\mathbf{u}+\mathbf{v} / 2)\right] .
\end{aligned}
$$

The condensate band operator $L_{C}$ is given in terms of the condensate band Hamiltonian

$H_{C}=\int \mathrm{d}^{3} \mathbf{x} \phi^{\dagger}(\mathbf{x})\left(-\frac{\hbar^{2} \nabla^{2}}{2 m}+V(\mathbf{x}, t)\right) \phi(\mathbf{x})+\frac{u}{2} \int \mathrm{d}^{3} \mathbf{x} \phi^{\dagger}(\mathbf{x}) \phi^{\dagger}(\mathbf{x}) \phi(\mathbf{x}) \phi(\mathbf{x})$

as

$$
L_{C} \phi(\mathbf{x}) \equiv\left[\phi(\mathbf{x}), H_{C}\right]
$$

In principle, a mean field or forward scattering term could also be included in $H_{C}$, and would alter the effective potential. In what follows, this possibility has been accounted for by our inclusion of a general time-dependent perturbing potential in (11), into which such a term can be absorbed.

4 This corrects an extra minus sign in the defining equation (56) of [39].

5 This corrects a misprint in equation (59) of [39] wherein $L_{C}$ appeared in place of $-L_{C}$ in the second and third lines of the master equation equivalent to (31). 


\subsection{Simplified local theory}

The full non-local theory is difficult to work with analytically and numerically. Fortunately, the full complexity is often not necessary, particularly if we are primarily interested in the growth/loss terms which are the focus of the present paper.

3.3.1. Approximations. A simplified master equation which encapsulates much of the physics of condensate growth is found using the following approximations:

(i) Linearization. The exponential in the forward-backward relation (30) is truncated at first order in powers of $(\epsilon-\mu) / k_{B} T$. This is valid as long as the condensate band chemical potential is not significantly different from the noncondensate band chemical potential. This constraint can be satisfied for a wide range of temperatures, and will hold for many physical situations of interest - the resulting SGPE is a valid description provided the energy fluctuations between the two bands are small relative to $k_{B} T$.

(ii) Low condensate band energy. We ignore the condensate band energy during collisions with noncondensate atoms by approximating $G^{(+)}(\mathbf{x}, \mathbf{v}, \epsilon) \approx G^{(+)}(\mathbf{x}, \mathbf{v}, 0)$. This is valid when $\mu \ll E_{R}$.

(iii) Narrow momentum width. We neglect the condensate band momentum by making the approximation $\phi(\mathbf{u} \pm \mathbf{v} / 2) \approx \phi(\mathbf{u})$. This is justified because $G^{(+)}(\mathbf{u}, \mathbf{v}, 0)$ is sharply peaked about $\mathbf{v}=0$. Indeed, for sufficiently smooth $F(\mathbf{u}, \mathbf{K})$ it behaves like a deltafunction, ensuring momentum conservation.

(iv) Uniform thermal cloud. We treat the growth amplitude $G^{(+)}(\mathbf{u}, \mathbf{v}, 0)$ as spatially constant. This is the main simplifying approximation of the present work, and corresponds to the high temperature regime where the thermal cloud density is approximately uniform over the condensate band. The growth parameter becomes

$$
\gamma=\frac{1}{k_{B} T} \int \mathrm{d}^{3} \mathbf{v} G^{(+)}(0, \mathbf{v}, 0),
$$

and in the high temperature regime takes the simple form [13]

$$
\gamma=\frac{16 k_{B} T a^{3}}{\hbar u},
$$

which we will use in this paper.

3.3.2. Local master equation. Implementing these approximations gives the local master equation for condensate growth

$$
\begin{aligned}
\left.\dot{\rho}_{C}\right|_{\text {growth }}=\gamma k_{B} T \int \mathrm{d}^{3} \mathbf{x}\left\{\left[\left[\phi(\mathbf{x}), \rho_{C}\right], \phi^{\dagger}(\mathbf{x})\right]+\left[\phi(\mathbf{x}),\left[\rho_{C}, \phi^{\dagger}(\mathbf{x})\right]\right]\right\} \\
-\gamma \int \mathrm{d}^{3} \mathbf{x}\left\{\left[\left\{\left(\mu-L_{C}\right) \phi(\mathbf{x})\right\} \rho_{C}, \phi^{\dagger}(\mathbf{x})\right]+\left[\phi(\mathbf{x}), \rho_{C}\left\{\left(\mu+L_{C}\right) \phi^{\dagger}(\mathbf{x})\right\}\right]\right\} .
\end{aligned}
$$

It can be seen that, except for the parts of $L_{C} \phi(\mathbf{x})$ and $L_{C} \phi^{\dagger}(\mathbf{x})$ corresponding to S-wave collisions, all terms of this master equation are of Lindblad form which ensures positive definiteness of the dissipative evolution. However, it has been shown by Munro and Gardiner [50] that the main effect of such terms is to generate weak initial transients which have a negligible effect on the physical predictions of the theory. 


\subsection{The stochastic Gross-Pitaevskii equation}

The master equation for the condensate band is now mapped onto an equation of motion for the multimode Wigner distribution. Derivatives higher than second order in the fields are neglected in order to derive a genuine Fokker-Planck equation with positive definite diffusion matrix. This can then be mapped to a stochastic differential equation for the complex condensate band field $\alpha(\mathbf{x}, t)$ [51]. We can now make a connection with section 2 where the operators $L_{\mathrm{GP}}$ and $\mathcal{P}$ were introduced in the context of the GPE and PGPE theory. Loosely speaking, there is a correspondence between the field operator $\phi(\mathbf{x}, t)$ and the stochastic wavefunction $\alpha(\mathbf{x}, t)$, and this leads to a correspondence between the term $L_{C} \phi(\mathbf{x}, t)$ in the master equation (36) and $L_{\mathrm{GP}} \alpha(\mathbf{x}, t)$ in the SGPE. In complete detail, one finds that the projector $\mathcal{P}$ also has a part to play in the SGPE, as is to be expected, since according to (27) we are working with a projected quantum field operator for the condensate band.

We eventually obtain the SGPE in the form of the Langevin field equation

$\mathrm{d} \alpha(\mathbf{x}, t)=-\frac{\mathrm{i}}{\hbar} \mathcal{P} L_{\mathrm{GP}} \alpha(\mathbf{x}, t) \mathrm{d} t+\gamma \mathcal{P}\left(\mu-L_{\mathrm{GP}}\right) \alpha(\mathbf{x}, t) \mathrm{d} t+\mathrm{d} W_{G}(\mathbf{x}, t)$,

where the noise is a vector Wiener process which satisfies

$$
\begin{aligned}
& \mathrm{d} W_{G}(\mathbf{x}, t) \mathrm{d} W_{G}\left(\mathbf{x}^{\prime}, t\right)=\mathrm{d} W_{G}^{*}(\mathbf{x}, t) \mathrm{d} W_{G}^{*}\left(\mathbf{x}^{\prime}, t\right)=0 \\
& \mathrm{~d} W_{G}^{*}(\mathbf{x}, t) \mathrm{d} W_{G}\left(\mathbf{x}^{\prime}, t\right)=2 \gamma k_{B} T \delta_{C}\left(\mathbf{x}, \mathbf{x}^{\prime}\right) \mathrm{d} t .
\end{aligned}
$$

This simple growth SGPE describes a finite temperature BEC in contact with a noncondensed thermal cloud and takes the form of the PGPE given in (25), with additional dissipation and noise terms.

\subsection{Continuity}

Using (37) and averaging over the noise using Itô rules leads to the continuity equation

$$
\begin{aligned}
\frac{\partial n_{\mathrm{GP}}(\mathbf{x})}{\partial t}+\nabla & \cdot \mathbf{j}_{\mathrm{GP}}(\mathbf{x})=\frac{1}{\hbar} 2 \operatorname{Im}\left\langle\mathcal{Q}^{*}\left\{\left(\delta V(\mathbf{x}, t)+u|\alpha(\mathbf{x})|^{2}\right) \alpha^{*}(\mathbf{x})\right\} \alpha(\mathbf{x})\right\rangle_{W} \\
& +\gamma 2 \operatorname{Re}\left\langle\mathcal{Q}^{*}\left\{\left[\delta V(\mathbf{x}, t)+u|\alpha(\mathbf{x})|^{2}\right] \alpha^{*}(\mathbf{x})\right\} \alpha(\mathbf{x})\right\rangle_{W} \\
& +\gamma 2 \operatorname{Re}\left\langle\mu|\alpha(\mathbf{x})|^{2}-\alpha^{*}(\mathbf{x}) L_{\mathrm{GP}} \alpha(\mathbf{x})\right\rangle_{W}
\end{aligned}
$$

where \langle\rangle$_{W}$ denotes the average over different realizations of the noise, and

$$
\begin{aligned}
\mathbf{j}_{\mathrm{GP}}(\mathbf{x}) & =\frac{\mathrm{i} \hbar}{2 m}\left\langle\alpha(\mathbf{x}) \nabla \alpha^{*}(\mathbf{x})-\alpha^{*}(\mathbf{x}) \nabla \alpha(\mathbf{x})\right\rangle_{W} \\
n_{\mathrm{GP}}(\mathbf{x}) & =\left\langle\alpha^{*}(\mathbf{x}) \alpha(\mathbf{x})\right\rangle_{W} .
\end{aligned}
$$

When there is no damping $(\gamma=0)$ the continuity equation for the resulting PGPE has an additional source term (the first term on the right-hand side of equation (40)). Note, however, that for any function $f(\mathbf{x})$ and projected wavefunction $\alpha(\mathbf{x}) \equiv \mathcal{P} \alpha(\mathbf{x})$

$$
\int \mathrm{d}^{3} \mathbf{x}\left[\mathcal{Q}^{*} f(\mathbf{x})\right] \alpha(\mathbf{x})=\int \mathrm{d}^{3} \mathbf{x} f(\mathbf{x}) \mathcal{Q} \alpha(\mathbf{x})=0,
$$

and consequently the source conserves atom number, simply redistributing the field.

\section{Finite temperature Ehrenfest relations}

The derivation proceeds in much the same way as the calculation of the standard Ehrenfest relations for the GPE, except that we make use of the identities for the $\mathcal{Q}$ projector (20)-(22), 
to eliminate terms where possible, and use Itô rules to determine the effect of thermal fluctuations. In the equation of motion for an arbitrary operator $\bar{A}$ the Itô correction comes about from the third term in the expression for the differential

$$
\mathrm{d}(\bar{A})=\int \mathrm{d}^{3} \mathbf{x} \alpha^{*}(\mathbf{x}) A \mathrm{~d} \alpha(\mathbf{x})+\mathrm{d} \alpha^{*}(\mathbf{x}) A \alpha(\mathbf{x})+\mathrm{d} \alpha^{*}(\mathbf{x}) A \mathrm{~d} \alpha(\mathbf{x})
$$

which is first order in $\mathrm{d} t$. The fluctuation terms then have a generic form that can be written as a trace over an appropriate projected operator.

\subsection{SGPE Ehrenfest relations}

Carrying out the spatial and stochastic averaging leads to the Ehrenfest relations for the SGPE

$$
\begin{aligned}
& \frac{\mathrm{d}\langle\overline{\mathbf{x}}\rangle_{W}}{\mathrm{~d} t}=\frac{\langle\overline{\mathbf{p}}\rangle_{W}}{m}+2 \gamma \operatorname{Re}\left\langle\overline{\mathbf{x}\left(\mu-L_{\mathrm{GP}}\right)}\right\rangle_{W}+2 \gamma k_{B} T \operatorname{tr} \mathcal{P} \mathbf{x}+Q_{\mathbf{x}} \\
& \frac{\mathrm{d}\langle\overline{\mathbf{p}}\rangle_{W}}{\mathrm{~d} t}=-\langle\overline{\nabla V(\mathbf{x}, t)}\rangle_{W}+2 \gamma \operatorname{Re}\left\langle\overline{\mathbf{p}\left(\mu-L_{\mathrm{GP}}\right)}\right\rangle_{W}+2 \gamma k_{B} T \operatorname{tr} \mathcal{P} \mathbf{p}+Q_{\mathbf{p}} \\
& \frac{\mathrm{d}\langle\overline{\mathbf{L}}\rangle_{W}}{\mathrm{~d} t}=-\frac{\mathrm{i}}{\hbar}\langle\overline{\mathbf{L} V(\mathbf{x}, t)}\rangle_{W}+\gamma 2 \operatorname{Re}\left\langle\overline{\mathbf{L}\left(\mu-L_{\mathrm{GP}}\right)}\right\rangle_{W}+2 \gamma k_{B} T \operatorname{tr} \mathcal{P} \mathbf{L}+Q_{\mathbf{L}} \\
& \frac{\mathrm{d}\left\langle H_{\mathrm{GP}}\right\rangle_{W}}{\mathrm{~d} t}=\overline{\left.\frac{\partial \delta V(\mathbf{x}, t)}{\partial t}\right\rangle_{W}}+2 \gamma\left\langle\overline{L_{\mathrm{GP}}\left(\mu-L_{\mathrm{GP}}\right)}\right\rangle_{W}+2 \gamma k_{B} T\left\langle\operatorname{tr} \mathcal{P} L_{\mathrm{GP}}\right\rangle_{W} \\
& \frac{\mathrm{d}\left\langle N_{\mathrm{GP}}\right\rangle_{W}}{\mathrm{~d} t}=2 \gamma\left\langle\overline{\mu-L_{\mathrm{GP}}}\right\rangle_{W}+2 \gamma k_{B} T \operatorname{tr} \mathcal{P},
\end{aligned}
$$

where the projector terms take the form

with

$$
Q_{A}=\frac{1}{\hbar} 2 \operatorname{Im}\left\{\left\langle\overline{F_{A}}\right\rangle_{W}\right\}+\gamma 2 \operatorname{Re}\left\{\left\langle\overline{F_{A}}\right\rangle_{W}\right\},
$$

$$
F_{A} \alpha(\mathbf{x}) \equiv\left(\delta V(\mathbf{x}, t)+u|\alpha(\mathbf{x})|^{2}\right) \mathcal{Q}[A \alpha(\mathbf{x})],
$$

and $A \in\{\mathbf{x}, \mathbf{p}, \mathbf{L}\}$. These equations are our main result. They serve as useful consistency conditions for numerical simulations of the SGPE and PGPE, and extend the intuitive Ehrenfest results to finite temperature. The Ehrenfest behaviour familiar from the GPE is modified by dissipation and fluctuation terms, and by the boundary corrections generated by the energy cutoff.

\subsection{Discussion}

4.2.1. Trace terms. A typical trace term is, for example,

$$
\operatorname{tr} \mathcal{P} \mathbf{x}=\overline{\sum_{n}} \int \mathrm{d}^{3} \mathbf{x} Y_{n}^{*}(\mathbf{x}) \mathbf{x} Y_{n}(\mathbf{x})
$$

so that the projected operators drive the condensate band. The nonlinear part of the driving term in the energy equation (48) is

$$
\begin{aligned}
\langle\operatorname{tr} \mathcal{P} u \mid \alpha\rangle\langle\alpha \mid\rangle_{W} & =\int \mathrm{d} \mathbf{x} \int \mathrm{d} \mathbf{y} u \delta(\mathbf{x}-\mathbf{y})\left\langle\alpha^{*}(\mathbf{x}) \alpha(\mathbf{y})\right\rangle_{W} \overline{\sum_{n}} Y_{n}(\mathbf{x}) Y_{n}^{*}(\mathbf{y}) \\
& =\int \mathrm{d} \mathbf{x} u\left\langle\alpha^{*}(\mathbf{x}) \alpha(\mathbf{x})\right\rangle_{W} \delta_{C}(\mathbf{x}, \mathbf{x}) .
\end{aligned}
$$

where $|\alpha\rangle\langle\alpha|$ is the pure state density operator for the condensate band field. This term arises from the equiposition commutator $\left[\phi(\mathbf{x}), \phi^{\dagger}(\mathbf{x})\right]=\delta_{C}(\mathbf{x}, \mathbf{x})$ which has a position-dependent finite value in the projected theory. 
4.2.2. Projector terms. Although all explicit projectors have been accounted for, the spatial integrals generate implicit projection since we are working with a projected stochastic wavefunction $\alpha \equiv \mathcal{P} \alpha$. It is immediately apparent from (51) that the boundary terms vanish when either $\left[A, H_{0}\right]=0$, or

$$
\mathcal{Q}\left[\left(\delta V(\mathbf{x}, t)+u|\alpha(\mathbf{x})|^{2}\right) \alpha(\mathbf{x})\right]=0,
$$

which is automatically true if the modes at the energy cutoff are weakly occupied. However, since the classical field method has been used we have assumed that all modes in the condensate band are significantly occupied. These two conditions can be reconciled if the occupation of the modes at the cutoff is small relative to the other modes in the system but still large enough to make the third-order derivatives in the equation of motion for the Wigner distribution unimportant.

\subsection{Special cases}

Equation (37) can be used to recover two useful equations which have been used to describe a number of interesting finite temperature effects in Bose-Einstein condensates including equilibrium properties and vortex lattice nucleation [21, 35-37, 45, 52].

4.3.1. The Gardiner-Anglin-Fudge equation. The Gardiner-Anglin-Fudge (GAF) equation $[38,52]$ is found from (37) by dropping the noise and setting the projector to the identity, to give

$$
\mathrm{i} \hbar \frac{\partial \alpha(\mathbf{x}, t)}{\partial t}=L_{\mathrm{GP}} \alpha(\mathbf{x}, t)+\mathrm{i} \hbar \gamma\left(\mu-L_{\mathrm{GP}}\right) \alpha(\mathbf{x}, t) .
$$

This describes a condensate in contact with a noise-free thermal cloud at chemical potential $\mu$. An equation of essentially the same form has been used by Penckwitt et al [52] to investigate vortex lattice formation ${ }^{6}$.

Combining (48) and (49) for the case where $\delta \dot{V}=0$, and where we neglect the noise, leads to

$$
\frac{\partial\left(H_{\mathrm{GP}}-\mu N_{\mathrm{GP}}\right)}{\partial t}=-2 \gamma \int \mathrm{d}^{3} \mathbf{x}\left|\left(\mu-L_{\mathrm{GP}}\right) \alpha(\mathbf{x}, t)\right|^{2},
$$

which is a monotonically decreasing function of time. Thus in the long time limit we recover a ground state of the GPE with chemical potential $\mu$.

4.3.2. The projected Gross-Pitaevskii equation. Putting $\gamma=0$, we recover the PGPE

$$
\mathrm{i} \hbar \frac{\partial \alpha(\mathbf{x}, t)}{\partial t}=\mathcal{P} L_{\mathrm{GP}} \alpha(\mathbf{x}, t) .
$$

Since the same equations of motion for energy and number hold for the PGPE, (57) also shows that the long time limit of

$$
\mathrm{i} \hbar \frac{\partial \alpha(\mathbf{x}, t)}{\partial t}=\mathcal{P} L_{\mathrm{GP}} \alpha(\mathbf{x}, t)+\mathrm{i} \hbar \gamma \mathcal{P}\left(\mu-L_{\mathrm{GP}}\right) \alpha(\mathbf{x}, t) .
$$

generates a ground state solution of the PGPE with chemical potential $\mu$. In the remainder of this paper, we consider two simple applications of the formalism developed above.

6 Although we note that numerical integration of (56) will lead to damping for any $\gamma$, rather than the small $\gamma$ limit required for the GAF equation of [39]. 


\section{Application to PGPE simulations of the Kohn mode: controlling artefacts}

One of the main aims of the classical field method is to deal with finite temperature BECs, but the method is constrained by the requirement that all modes in the condensate band are highly occupied. It is clear that if there is significant occupation near the cutoff the dynamics can be radically altered, but it is not sufficient to simply monitor the occupation numbers. It is preferable to find a strict dynamical criterion that ensures the validity of the simulations. Rather than tackle this general problem at finite temperature, in this paper we will simply show that at zero temperature it is possible to use the Ehrenfest relations to construct a reliable estimator of the error arising from the cutoff for a particular type of motion: the Kohn mode oscillation.

To consider a simple example of the phase space boundary effects (given by $Q_{A}$ in (45)-(49)) we consider a one-dimensional (1D) model consisting of a harmonically trapped partially condensed Bose gas. We also take $\gamma \equiv T \equiv 0$ so that $\dot{N}_{\mathrm{GP}} \equiv \dot{H}_{\mathrm{GP}} \equiv 0$, and ${ }^{7}$

$$
\begin{aligned}
\mathrm{i} \frac{\partial \alpha}{\partial t} & =\mathcal{P}\left(\frac{1}{2}\left(-\frac{\partial^{2}}{\partial x^{2}}+x^{2}\right) \alpha+\lambda|\alpha|^{2} \alpha\right) \\
\frac{\mathrm{d} \bar{x}}{\mathrm{~d} t} & =\bar{p}+Q_{x} \\
\frac{\mathrm{d} \bar{p}}{\mathrm{~d} t} & =-\bar{x}+Q_{p},
\end{aligned}
$$

where $\lambda$ is the dimensionless interaction strength. The details of how this relates to anisotropic trap geometry will not concern us here. We will simply consider modest effective nonlinearities of order $10<\lambda N_{\mathrm{GP}}<1000$ to determine the validity of the criterion developed below. The energy cutoff takes the form

$$
E_{R}=\bar{N}+\frac{1}{2}
$$

where $\bar{N}$ is the energy quantum number of the highest single particle state in the condensate band.

\subsection{Projector terms in the harmonic oscillator basis}

To evaluate the projector terms we require some properties of the harmonic oscillator eigenstates. In terms of the Hermite polynomials $H_{n}(x)$, the eigenstates of the single particle Hamiltonian

$$
Y_{n}(x)=\frac{H_{n}(x)}{\pi^{1 / 4} \sqrt{2^{n} n !}} \mathrm{e}^{-x^{2} / 2}
$$

are coupled by the $x$ and $p$ operators to

$$
\begin{aligned}
& x Y_{n}(x)=\frac{1}{\sqrt{2}}\left(\sqrt{n} Y_{n-1}(x)+\sqrt{n+1} Y_{n+1}(x)\right) \\
& p Y_{n}(x)=\frac{\mathrm{i}}{\sqrt{2}}\left(\sqrt{n+1} Y_{n+1}(x)-\sqrt{n} Y_{n-1}(x)\right),
\end{aligned}
$$

so that the projector generates the terms

$$
\mathcal{Q} x \alpha(x, t)=\sqrt{\frac{\bar{N}+1}{2}} \alpha_{\bar{N}}(t) Y_{\bar{N}+1}(x)
$$

7 We work in units of $x_{0}=(\hbar / m \omega)^{1 / 2}, t_{0}=\omega^{-1}$ and $k_{0}=1 / x_{0}$ for length and time and wave vector respectively. 


$$
\mathcal{Q} p \alpha(x, t)=\mathrm{i} \sqrt{\frac{\bar{N}+1}{2}} \alpha_{\bar{N}}(t) Y_{\bar{N}+1}(x),
$$

and the equations of motion are

$$
\begin{aligned}
& \frac{\mathrm{d} \bar{x}}{\mathrm{~d} t}=\bar{p}+\operatorname{Re}\{g(\bar{N})\} \\
& \frac{\mathrm{d} \bar{p}}{\mathrm{~d} t}=-\bar{x}+\operatorname{Im}\{g(\bar{N})\},
\end{aligned}
$$

where

$$
g(n)=\lambda \mathrm{i} \sqrt{2(n+1)} \alpha_{n}^{*}(t) \int \mathrm{d} x Y_{n+1}^{*}(x)|\alpha(x, t)|^{2} \alpha(x, t) .
$$

The projector correction is essentially a boundary effect caused by the nonlinear term of the GPE. This is to be expected since it is the only term in the PGPE that can cause transitions between states in the condensate and noncondensate bands. Since $g(\bar{N})$ is proportional to $\alpha_{\bar{N}}^{*}(t)$, weak occupation near the cutoff will naturally give Ehrenfest evolution. However, it is already apparent from the appearance of the nonlinear term that this condition alone is not sufficient to guarantee validity.

A useful measure is the relative error arising from the term $g(\bar{N})$ in the above equations. Since the Ehrenfest equations involve easily computed averages, one could simply monitor the ratios $|\operatorname{Re}\{g(\bar{N})\} / \bar{p}|$ and $|\operatorname{Im}\{g(\bar{N})\} / \bar{x}|$. Unfortunately, these are unsuitable for this purpose because $\bar{x}$ and $\bar{p}$ periodically traverse the origin.

\subsection{Phase space picture}

The rotational symmetry of the cutoff in phase space may be exploited by using the complex phase space coordinate $\bar{z} \equiv \bar{x}+\mathrm{i} \bar{p}$, for which the equation of motion is

$$
\frac{\mathrm{d} \bar{z}}{\mathrm{~d} t}=-\mathrm{i} \bar{z}+g(\bar{N}) \text {. }
$$

Equation (72) gives a simple physical picture of the role of the cutoff energy in phase space expressed in terms of the overlap integral (71). This suggests that the ratio

$$
E_{z}=\left|\frac{g(\bar{N})}{\bar{z}}\right|
$$

may be used to estimate the size of artefacts introduced by the cutoff.

This will be demonstrated using the following numerical test: we evolve a ground state solution of the PGPE for different initial momenta. For small momentum kicks we find undamped Kohn mode oscillations, confirming that the projector has no effect on the dynamics. Above some (yet to be determined) kick strength the shape oscillations arising from the projector are no longer negligible. At the critical kick strength, the edge of the condensate reaches the boundary of the available phase space. In position space this occurs when the condensate reaches the semi-classical turning point of the highest energy mode in the condensate band. The projector then comes into play, ensuring that the condensate wavefunction cannot make radial excursions in phase space that exceed the cutoff energy. This generates shape oscillations which become rather violent for a large kick strength.

A more informative picture of the process is found by transforming to classical phase space (which is detailed in the appendix). Snapshots of the modulus of the single particle Wigner function

$$
W(x, k, t)=\frac{1}{2 \pi} \int \mathrm{d} y \mathrm{e}^{\mathrm{i} k y} \alpha^{*}(x+y / 2, t) \alpha(x-y / 2, t)
$$




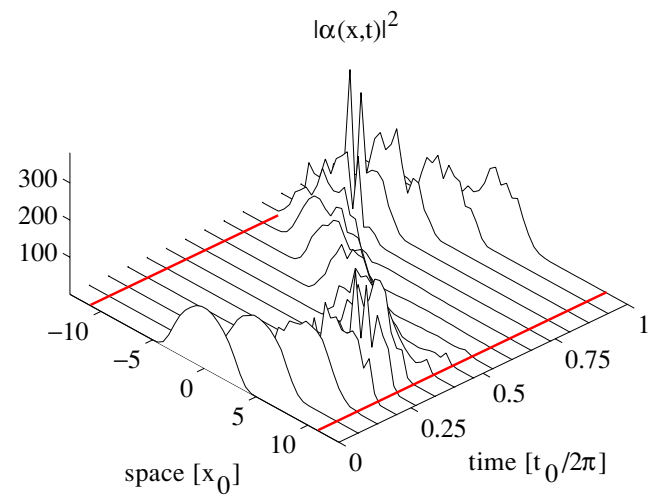

(a) Density time series

$$
\log (1+|\mathrm{W}(\mathrm{x}, \mathrm{k})|)
$$

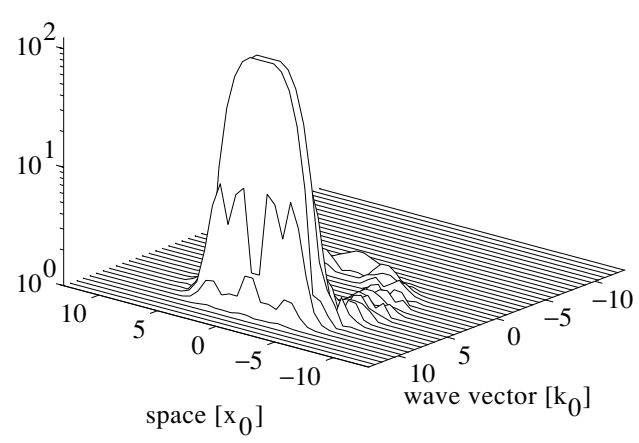

(c) Initial state shifted to $k_{0}=8$. $\log (1+|\mathrm{W}(\mathrm{x}, \mathrm{k})|)$

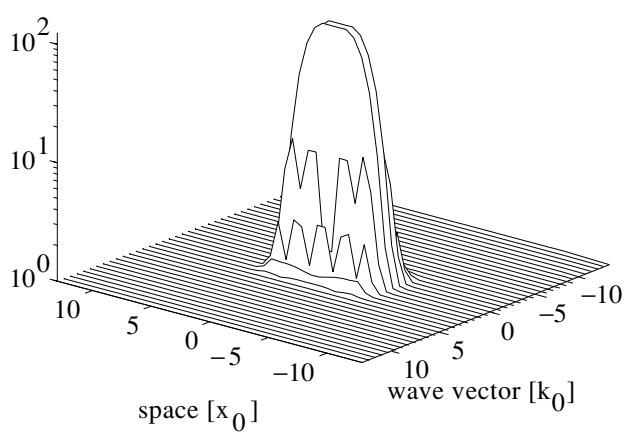

(b) PGPE ground state

$$
\log (1+|\mathrm{W}(\mathrm{x}, \mathrm{k})|)
$$

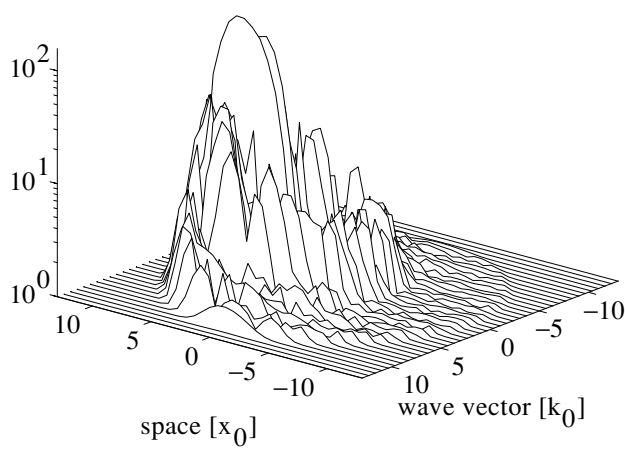

(d) State after $1 / 4$ trap period

Figure 2. A simulation used to determine the threshold value of $E_{z}$, defined in (75), for a ground state wavefunction of the PGPE. (a) Distorted Kohn mode for motion near the edge of the available space (the semi-classical turning point of the cutoff mode, given by the solid line at $x= \pm 12 x_{0}$ ). (b) Wigner function for the initial ground state solution of the GAF equation with nonlinear constant $\tilde{u} N=100$, cutoff $\bar{N}=60$. (c) Projected initial state after a shift in momentum space to wave vector $k_{0}=8$. (d) The wavefunction has filled the available phase space after $1 / 4$ of a trap period.

reveal the role of the projector, and the results for a high momentum simulation exhibiting distorted Kohn mode oscillations are shown in figure 2. It should be stressed here that although the initial momentum kick has a negligible effect on the shape of the condensate, the condensate wavefunction soon fills the available phase space. This is shown in figure 2(d), and is indicative of the spurious thermalization that can be caused by the interplay between the cutoff and S-wave collisions.

\subsection{Numerical determination of the threshold for $E_{z}$}

The threshold at which $E_{z}$ becomes significant is now determined in two ways: we examine the dependence of $E_{z}$ on the cutoff at fixed nonlinearity as shown in table 1; secondly, we find the variation of $E_{z}$ with nonlinearity for a given cutoff as shown in table 2 . In each table we show the values at which there is appreciable (of order $\sim 1 \%$ ) change to the time dependence of $\bar{x}$ and $\bar{p}$. It appears that we can ensure that the cutoff is not significantly altering the dynamics 
Table 1. Threshold values of $k_{0}$ and $E_{z}$ for a range of cutoff energies. Other values are $\lambda N_{\mathrm{GP}}=$ $170, \mu=20$.

\begin{tabular}{lll}
\hline Cutoff $\bar{N}$ & $k_{0}$ & $\max \left[E_{z}\right] \times 10^{4}$ \\
\hline 30 & 1 & 20 \\
40 & 2.2 & 10 \\
50 & 2.5 & 2 \\
60 & 4 & 2 \\
100 & 7.3 & 1 \\
\hline
\end{tabular}

Table 2. Threshold values of $k_{0}$ and $E_{z}$ for a range of nonlinear constants, with fixed cutoff $\bar{N}=100$.

\begin{tabular}{clc}
\hline Nonlinearity $\lambda N_{\mathrm{GP}}$ & $k_{0}$ & $\max \left[E_{z}\right] \times 10^{4}$ \\
\hline 59 & 9 & 2 \\
168 & 6.7 & 2 \\
476 & 4.8 & 8 \\
766 & 3.3 & 8 \\
1100 & 2.2 & 10 \\
\hline
\end{tabular}

by requiring

$$
E_{z}<10^{-4}
$$

It is important to note that, more generally, when any dynamical simulation is considered there will be a dominant set of moments that encapsulate the dynamics, and it is the effect of the projector on these quantities that must be considered. Higher order equations of the Ehrenfest type could then be used to extend the method we have described here.

\section{Application to PGPE simulations of the thermal Bose gas: the optimal plane wave representation}

The classical field method requires that the majority of modes used in the simulation of a Bose gas are highly occupied. When imposing this condition consistently near equilibrium in a harmonic trap this requires that a strict energy cutoff should be used. Such a cutoff is best implemented in the basis of harmonic oscillator eigenstates, since, in this basis, the full interacting Hamiltonian for the finite temperature system is approximately diagonal at the cutoff energy. Since the plane wave basis is often used for classical field simulations of trapped systems it is important to evaluate the validity of such a procedure against the more accurate procedure based on an exact energy cutoff.

We can use projected Ehrenfest relations to get an idea of the kinds of artefacts that arise when using the plane wave basis to represent a trapped system at finite temperature.

\subsection{Plane wave basis}

6.1.1. Modelling a homogeneous condensate with a plane wave basis. We write the wavefunction in terms of the basis of plane waves with periodic boundary conditions as

$$
\alpha(x, t)=\sum_{k=-K}^{K} \alpha_{k}(t) \frac{\mathrm{e}^{\mathrm{i} k x}}{\sqrt{L}}
$$


where $K$ is related to the spatial span $L$ according to $K=2 \pi N_{c} / L$. From this we have

$$
\mathcal{Q}(x \alpha)=\mathcal{Q} \sum_{k=-K}^{K} \alpha_{k}(t) \frac{-\mathrm{i}}{\sqrt{L}} \frac{\partial}{\partial k} \mathrm{e}^{\mathrm{i} k x},
$$

which we can approximate by

$$
\mathcal{Q}(x \alpha) \approx \mathcal{Q} \sum_{k=-K}^{K} \alpha_{k}(t) \frac{-\mathrm{i}}{\sqrt{L}} \frac{\mathrm{e}^{\mathrm{i}(k+\Delta) x}-\mathrm{e}^{\mathrm{i}(k-\Delta) x}}{2 \Delta},
$$

when the momentum grid spacing $\Delta$ is small. This leads to

$$
\mathcal{Q}(x \alpha) \approx \frac{-\mathrm{i}}{2 \Delta \sqrt{L}}\left[\alpha_{K}(t) \mathrm{e}^{\mathrm{i}(K+\Delta) x}-\alpha_{-K}(t) \mathrm{e}^{-\mathrm{i}(K+\Delta) x}\right] .
$$

Since the momentum operator commutes with the Hamiltonian and the potential is absent, the averages evolve according to $\dot{N}_{\mathrm{GP}}=\dot{H}_{\mathrm{GP}}=\dot{\bar{p}}=0$ and

$$
\frac{\mathrm{d} \bar{x}}{\mathrm{~d} t}=\frac{\bar{p}}{m}+Q_{x}(K)+S_{\sigma}(L)
$$

where

$Q_{x}(K)=\frac{u}{\hbar} 2 \operatorname{Im}\left\{\int \mathrm{d} x|\alpha(x, t)|^{2} \alpha(x, t)^{*} \frac{-\mathrm{i}}{2 \Delta \sqrt{L}}\left[\alpha_{K}(t) \mathrm{e}^{\mathrm{i}(K+\Delta) x}-\alpha_{-K}(t) \mathrm{e}^{-\mathrm{i}(K+\Delta) x}\right]\right\}$,

$S_{\sigma}(L)=\frac{1}{2 m}\left[(x \alpha(x, t))\left(p \alpha^{*}(x, t)\right)-\alpha^{*}(x, t)(p x \alpha(x, t))\right]_{-L / 2}^{L / 2}$.

The boundary term $S_{\sigma}$ arises from the finite span of the periodic basis and does not arise in the use of the harmonic trap basis where the basis elements are defined over all space. For the sake of formal clarity, we have omitted terms of this type in our derivation of the generalized Ehrenfest relations in section 4, but they will be important for the special case where $\alpha(\mathbf{x}, t)$ is non-zero at the box boundaries_-as can occur in a periodic description.

6.1.2. Modelling a harmonically trapped condensate with a plane wave basis. We have seen that there is a boundary term for the $\dot{\bar{x}}$ equation when the plane wave basis is used for the case where $V(x, t)=0$. When the plane wave basis is used to model a system confined by a harmonic potential, the results follow from (51) by putting $\delta V(x)=m \omega^{2} x^{2} / 2$, so that the entire potential becomes a variation with respect to $H_{0}$. The continuity equation is now given by

$$
\frac{\partial n_{\mathrm{GP}}(\mathbf{x})}{\partial t}+\nabla \cdot \mathbf{j}_{\mathrm{GP}}(\mathbf{x})=\frac{2}{\hbar} \operatorname{Im}\left(\mathcal{Q}^{*}\left[\left(m \omega^{2} x^{2} / 2+u|\alpha|^{2}\right) \alpha^{*}\right] \alpha\right),
$$

and the Ehrenfest relations are

$$
\begin{aligned}
\frac{\mathrm{d} \bar{x}}{\mathrm{~d} t} & =\frac{\bar{p}}{m}+Q_{x}(K) \\
\frac{\mathrm{d} \bar{p}}{\mathrm{~d} t} & =-m \omega^{2} \bar{x},
\end{aligned}
$$

with the boundary term given by

$$
\begin{aligned}
Q_{x}(K)=\frac{1}{\hbar} 2 & \operatorname{Im}\left(\int \mathrm{d} x\left(m \omega^{2} x^{2} / 2+u|\alpha(x, t)|^{2}\right) \alpha^{*}(x, t)\right. \\
& \left.\times \frac{-\mathrm{d}}{2 \Delta \sqrt{L}}\left[\alpha_{K}(t) \mathrm{e}^{\mathrm{i}(K+\Delta) x}-\alpha_{-K}(t) \mathrm{e}^{-\mathrm{i}(K+\Delta) x}\right]\right) .
\end{aligned}
$$


We have neglected $S_{\sigma}$ because the trapping potential will ensure that the wavefunction is negligible at the grid edge for a well chosen basis. Comparing the plane wave basis equations (84)-(86) with the trap basis results (69)-(71) (for $\delta V \equiv 0$ ), we see that there are two notable differences: (i) the boundary corrections occur in both the $\dot{\bar{x}}$ and $\dot{\bar{p}}$ equations for the trap basis, and only in the $\dot{\bar{x}}$ equation in the plane wave basis; and (ii) there is a contribution from the potential in the boundary correction for $\dot{\bar{x}}$ for the plane wave basis. This is particularly significant since this term can potentially assume large values when $\alpha_{K}(t) \neq 0$, even in the linear regime. This raises the question of how to minimize such boundary effects, which we now address.

\subsection{The optimal plane wave representation}

There is a certain degree of freedom in choosing a plane wave basis for representing a harmonically trapped system. Here we show how to obtain the optimal plane wave basis that best captures the lowest harmonic oscillator states. We would expect this to be the best plane wave representation for modelling harmonically trapped systems.

We consider a basis of $\bar{N}$ plane wave states taken to extend over the spatial box of size $x \in[-L / 2, L / 2]$, as defined in (76). For fixed $\bar{N}$, the only free parameter in constructing the plane wave basis is $L$. Making $L$ large is done at the expense of decreasing the momentum width that can be represented on the grid, while conversely decreasing $L$ limits the spatial extent of the system, but increases the momentum range.

Here we give a simple argument for an optimal choice of $L$ at fixed $\bar{N}$. In harmonic oscillator units the single particle Hamiltonian for the harmonic oscillator takes the form

$$
\bar{H}=\frac{1}{2} \bar{k}^{2}+\frac{1}{2} \bar{x}^{2},
$$

where bars are used to indicate dimensionless quantities (e.g., see section VB1 of [53]). In these units, the Hamiltonian and its eigenstates take the same form in coordinate and wave vector space. So the best grid choice will be when our numerical grids for (dimensionless) position and wave vector space are identical, i.e., when $\bar{L}=\bar{K}$. Returning to dimensioned units this optimal choice is

$$
L_{\mathrm{opt}}=\sqrt{\frac{2 \pi \hbar \bar{N}}{m \omega}} .
$$

From this expression we obtain directly the largest momentum that can be represented on the grid, which is given by $K_{\text {opt }}=\pi \bar{N} / L_{\text {opt }}$ (i.e., the limits of the sum in (76)).

To quantify the sensitivity to non-optimal choices of $L$, we show in figure 3 the spectrum of energies found by diagonalizing the harmonic oscillator Hamiltonian (87) in the plane wave basis for a range of $L$ values. These results show that $L_{\mathrm{opt}}$ is clearly the best choice, however even for $L=L_{\mathrm{opt}}$ only about half the eigenstates are accurately obtained.

\subsection{Comparison of plane wave and harmonic oscillator phase space}

The harmonic oscillator and plane wave bases differ somewhat in the regions of phase space they represent. This difference is reflected in the position quadrature grids associated with each basis, shown in figure 4(a). It is apparent from this figure that the spacing between quadrature points of the harmonic oscillator basis varies from being dense in the central region to sparse at the edges. This enables the basis to capture large momentum states (i.e., fast spatial variations) at small displacements from the trap centre, and smaller momentum states at large displacements. This suggests that for fixed $\bar{N}$, the harmonic oscillator basis captures 


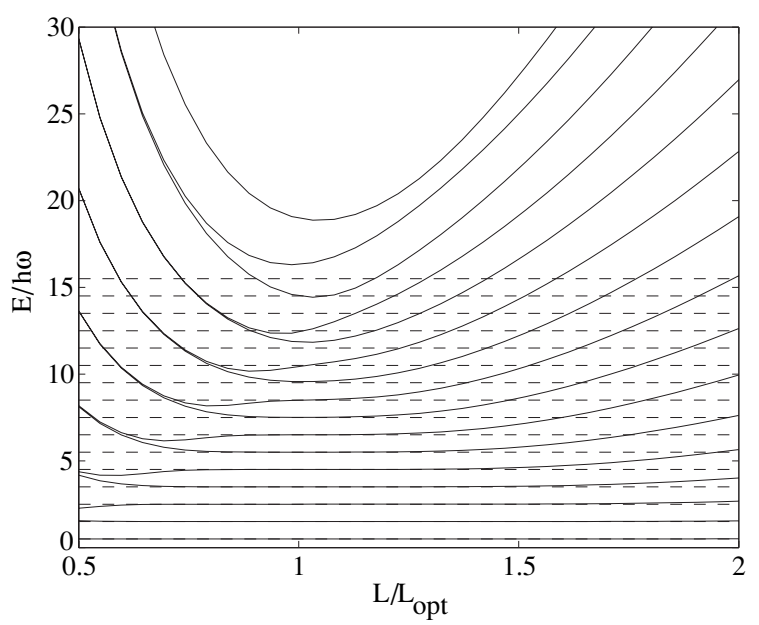

Figure 3. The numerical spectrum of the harmonic oscillator Hamiltonian. The solid lines are the plane wave results found by diagonalizing the harmonic oscillator Hamiltonian on a grid of $\bar{N}=16$ points of width $L$. The dashed lines indicate the 16 lowest energies of the exact eigenspectrum, which corresponds to (trivially) diagonalizing the Hamiltonian in the harmonic oscillator basis for $\bar{N}=16$.

$$
\begin{aligned}
& \text { (a) Harm. osc. grid }
\end{aligned}
$$

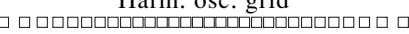

$$
\begin{aligned}
& \text { Plane wave grid (Lopt) } \\
& 000000000000000000000000000000 \\
& \text { position [arb. units] }
\end{aligned}
$$

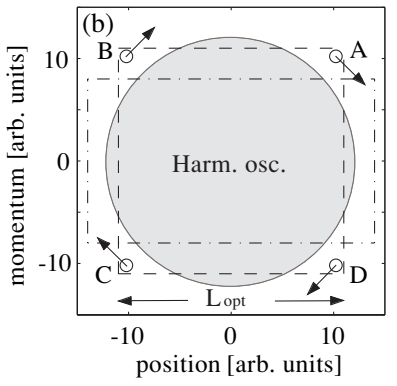

Figure 4. Phase space of the harmonic oscillator and plane wave representations. (a) The quadrature grids for the optimal plane wave basis (circles) and the harmonic oscillator basis (squares). (b) The approximate phase space captured by the optimal plane wave (dashed boundary), a non-optimal plane wave (dashed-dotted boundary), and harmonic oscillator (solid boundary) bases. The points $A-D$ indicate the evolution of anomalous trajectories (see text).

a circular region of phase space, as shown schematically in figure 4(b). In contrast, the plane wave grid is equally spaced over the entire region $[-L / 2, L / 2]$ (see figure 4(a)). This means the plane wave representation is equally well able to represent high momentum states at all displacements from the trap centre, suggesting that this basis captures a rectangular region of phase space (see figure 4(b)).

Classically, the motion of a harmonic oscillator corresponds to perfect circular trajectories in phase space, and we therefore expect that a circular phase space projector forms the ideal energy cutoff. We note that the optimal plane wave representation corresponds to choosing $L$ so that the maximum kinetic and potential energies associated with the edge values of the position and wave vector grids, respectively, are equal, i.e., $\frac{1}{2} m \omega^{2}\left(L_{\text {opt }} / 2\right)^{2}=\hbar^{2}\left(K_{\text {opt }} / 2\right)^{2} / 2 m$. For this case the phase space region bounded by the plane wave representations most closely matches the harmonic oscillator space (see the dashed line in figure 4(b)). In comparison, 

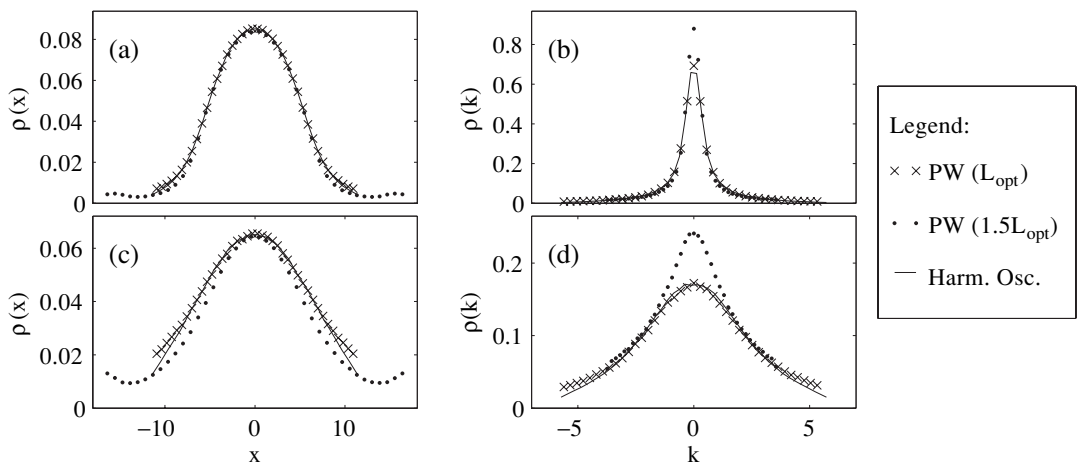

Figure 5. Results for the equilibrium position and momentum density profiles of a 1D thermal Bose gas. Low energy case: simulations for a total energy of $E=14 \hbar \omega$ (a) equilibrium position density, (b) equilibrium momentum density. High energy case: simulations for a total energy of $E=21 \hbar \omega$ (c) equilibrium position density, (d) equilibrium momentum density. Results are calculated by time-averaging classical field calculations carried out in the different bases under consideration: plane wave for $L=L_{\mathrm{opt}}, L=1.5 L_{\mathrm{opt}}$, and the harmonic oscillator basis. Other simulation parameters are $\lambda N=200, \bar{N}=40$.

a non-optimal plane wave basis has an energy projector that restricts the kinetic energy and potential energy inconsistently, giving rise to a rectangular phase space boundary (e.g., see the dashed-dotted line in figure 4(b)).

The high energy modes in the plane wave phase space exhibit anomalous dynamics arising from aliasing the region of phase space that is inconsistently represented. We illustrate this by examining the dynamics of a phase space point $A$ in figure 4(b). This point evolves along the trajectory indicated by the arrow until it reaches the right position boundary (dashed line). It is then aliased to the left position boundary and continues to evolve through point $B$, before reaching the upper momentum boundary. It will then pass through point $C, D$ and then return to $A$. The overall result is that states lying near the corners of the phase space region undergo a counterclockwise evolution through phase space, in contrast to the normal clockwise evolution through phase space. In application to the SGPE formalism, we would expect that system disturbances with momentum and position characteristics lying in these corner regions would evolve in this anomalous manner.

\subsection{Plane wave and harmonic oscillator representations of a thermalized Bose gas}

In this section, we compare the effect of basis on simulations of a harmonically trapped 1D gas using the PGPE equation (58). Our method follows the approach used by Davis et al in [36]: for each of the bases under consideration we evolve a randomized initial state of definite energy according to the PGPE. This evolution is expected to be ergodic, and by appropriately timeaveraging pure state expectations we are able to obtain ensemble averages. To compare the different bases we then examine the equilibrium position and momentum density distributions, as well as condensate fractions.

In detail, the simulations we have conducted are for a dimensionless interaction strength of $\lambda N=200$ for bases with 40 modes (i.e., $\bar{N}=40$ ). In figure 5 we present results for the density distributions found from evolving randomized initial states with a total energy of $E=14 \hbar \omega$ (figures 5(a) and (b)), and for $E=21 \hbar \omega$ (figures 5(c) and (d)). These two choices of energy correspond to a strongly condensed system (with a large condensate fraction) and a system close to the transition respectively (we will discuss condensate fraction later in this 
Table 3. Condensate fraction for a one-dimensional thermal Bose gas obtained using various numerical bases.

\begin{tabular}{lll}
\hline Basis & $\begin{array}{l}\text { Condensate fraction } \\
E=14 \hbar \omega\end{array}$ & $\begin{array}{l}\text { Condensate fraction } \\
E=21 \hbar \omega\end{array}$ \\
\hline Harmonic oscillator & 0.370 & 0.072 \\
PW $\left(0.5 L_{\text {opt }}\right)$ & 0.815 & 0.238 \\
PW $\left(0.8 L_{\text {opt }}\right)$ & 0.392 & 0.097 \\
PW $\left(1.0 L_{\text {opt }}\right)$ & 0.370 & 0.078 \\
PW $\left(1.2 L_{\text {opt }}\right)$ & 0.360 & 0.078 \\
PW $\left(1.5 L_{\text {opt }}\right)$ & 0.444 & 0.116 \\
\hline
\end{tabular}

section). The three bases we compare are the harmonic oscillator basis, and plane waves bases with $L=L_{\mathrm{opt}}, L=1.5 L_{\mathrm{opt}}$.

The results shown in figure 5 confirm that the the optimal plane wave basis is in better agreement with the trap basis than the non-optimal basis. A particular weakness of the plane wave representations is that density distributions in the wings tend to decrease more slowly than the trap basis. In the $L=1.5 L_{\mathrm{opt}}$ case the density distribution even begins to increase near the boundary, as is apparent in figures 5(a) and (c). This behaviour is most likely due to the inconsistent manner that the plane wave basis represents the highest energy states as discussed in the previous subsection. This will have serious implications for schemes that require the behaviour of the distribution wings to calculated temperature, and is likely to have affected the temperature calculations made in [31].

Finally, we examine how the condensate fraction is influenced by the choice of basis. We determine the condensate fraction using the Penrose-Onsager criterion [31, 54, 55]. To do this, we calculate the one-body density matrix by time-averaging the classical field, i.e., $\rho_{1 \mathrm{~B}}\left(x, x^{\prime}\right)=\left\langle\psi^{*}(x) \psi\left(x^{\prime}\right)\right\rangle_{\text {time ave. }}$. The condensate occupation is determined as the largest eigenvalue of the one-body density matrix:

$$
\int \mathrm{d} x^{\prime} \rho_{1 \mathrm{~B}}\left(x, x^{\prime}\right) \phi_{0}\left(x^{\prime}\right)=N_{0} \phi_{0}(x)
$$

The results are presented in table 3 for the cases considered in figure 5, augmented by results from a wider range of plane wave bases. These results show conclusively that non-optimal plane wave bases can have a dramatic influence on the physical properties of the system being simulated.

\section{Conclusions}

We have shown how to derive exact Ehrenfest relations for the SGPE, an equation of motion which has become an important tool in the study of finite temperature Bose-Einstein condensates, and contains the PGPE and GPE descriptions as special cases. We have pointed out the link between these approaches and investigated the effects of the energy cutoff and thermal fluctuations.

For BEC simulations using the PGPE or SGPE in the classical field approximation, the well-known validity condition requires that all modes in the condensate band are significantly occupied [21, 39, 42]. However, it is the relative occupation at the phase space boundary that determines the influence of the projector. Thus the cutoff can be chosen so that the projector does not generate spurious dynamics, even though the modes near the cutoff may have moderate occupation. However, this can be a delicate balance, and we have demonstrated 
the possibility of finding computable dynamical tests of the PGPE that are not reliant on simply monitoring the mode occupations.

The projector in the SGPE generates boundary terms that arise because the GrossPitaevskii time evolution can evolve the wavefunction outside the condensate band. This kind of evolution can arise through either the nonlinear term or from an additional potential which is not part of the single particle Hamiltonian used to generate the representation basis. It therefore becomes important to choose the right basis, and we have shown that using the plane wave basis for a harmonically trapped BEC in thermal equilibrium can significantly alter the equilibrium condensate fraction. However, if the spatial grid is chosen to optimally reproduce the single particle spectrum of the harmonic trap the error is minimized. This choice of grid also has a simple physical interpretation since it provides the optimal covering of the phase space region enclosed by the energy cutoff.

Future development of the SGPE theory will require a more complete description of the way a dynamically evolving noncondensate band interacts with the condensate band. The work presented here gives some insight into the form such a description must take.

\section{Acknowledgments}

AB thanks Murray Olsen, Matthew Davis, Piyush Jain and Adam Norrie for many useful comments. This work was supported by the Marsden Fund of the Royal Society of New Zealand contract PVT-202, and by the New Zealand Foundation for Research, Science and Technology contract TAD-1054 and the Australian Research Council.

\section{Appendix. Transforming to classical phase space}

In order to examine the behaviour of the condensate band wavefunction in classical phase space we require the connection between the mode representation $\alpha(x, t)=\sum_{n} \alpha_{n}(t) Y_{n}(x)$ and the Wigner function

$$
W(x, k, t)=\frac{1}{2 \pi} \int \mathrm{d} y \mathrm{e}^{\mathrm{i} k y} \alpha^{*}(x+y / 2, t) \alpha(x-y / 2, t)
$$

for a wavefunction expressed in the harmonic oscillator representation.

We insert the mode decomposition into (A.1) to get

$$
W(x, k, t)=\overline{\sum_{n}} \overline{\sum_{m}} \alpha_{n}^{*}(t) \alpha_{m}(t) W_{n m}(x, k),
$$

where the modes $Y_{n}(x)$ are the orthonormal eigenstates of the harmonic trap given by (64), and

$$
W_{n m}(x, k) \equiv \frac{1}{2 \pi} \int \mathrm{d} y \mathrm{e}^{\mathrm{i} k y} Y_{n}^{*}(x+y / 2) Y_{m}(x-y / 2) .
$$

For brevity we will use the notation $W_{n}^{q}(x, k)=W_{n, n+q}(x, k)$.

We make use of the contour representation of the Hermite polynomials [56] to write the modes as

$$
Y_{n}(x)=\frac{\mathrm{e}^{-x^{2} / 2}}{\sqrt{2^{n} n ! \sqrt{\pi}}} \oint \frac{\mathrm{d} t \mathrm{e}^{-t^{2}+2 t x}}{t^{n+1}}
$$

so that

$W_{n}^{q}(x, k)=\frac{\mathrm{e}^{-x^{2}}}{2 \pi(2 \pi \mathrm{i})^{2}} \sqrt{\frac{n !(n+q) !}{2^{2 n+q} \pi}} \oint \frac{\mathrm{d} s \mathrm{e}^{-s^{2}+2 s x}}{s^{n+q+1}} \oint \frac{\mathrm{d} t \mathrm{e}^{-t^{2}+2 t x}}{t^{n+1}} \int \mathrm{d} y \mathrm{e}^{-y^{2} / 4+y(\mathrm{i} k+t-s)}$ 
where the contours enclose the origin. Carrying out the $y$ and $t$ integrals, and then using the change of variables $s=z(x-\mathrm{i} k) /(z-1)$, we reach the expression

$W_{n}^{q}(x, k)=\frac{(-1)^{n} \mathrm{e}^{-x^{2}-k^{2}}}{\pi} \sqrt{\frac{n ! 2^{q}}{(n+q) !}}(x-\mathrm{i} k)^{q} \frac{1}{2 \pi \mathrm{i}} \oint \frac{\mathrm{d} z}{z^{n+1}} \frac{\mathrm{e}^{-2\left(x^{2}+k^{2}\right) z /(1-z)}}{(1-z)^{q+1}}$.

In this form, we can identify the generating function for the associated Laguerre polynomials [56]

$$
\frac{\mathrm{e}^{-x z /(1-z)}}{(1-z)^{q+1}}=\sum_{n=0}^{\infty} z^{n} L_{n}^{q}(x)
$$

to finally obtain

$$
W_{n}^{q}(x, k)=\frac{(-1)^{n}}{\pi} \sqrt{\frac{n ! 2^{q}}{(n+q) !}} \mathrm{e}^{-x^{2}-k^{2}}(x-\mathrm{i} k)^{q} L_{n}^{q}\left(2\left(x^{2}+k^{2}\right)\right) .
$$

When $q=0$ we recover the Wigner function for a number state which is a well-known result in quantum optics [51]. Using the symmetry $W_{n+q, n}(x, k)=W_{n}^{q}(x, k)^{*}$, an efficient transformation to phase space is given by

$W(x, k, t)=2 \operatorname{Re}\left\{\sum_{q=0}^{\bar{N}} \sum_{n=0}^{\bar{N}-q} \alpha_{n}^{*}(t) \alpha_{n+q}(t) W_{n}^{q}(x, k)\right\}-\sum_{n=0}^{\bar{N}}\left|\alpha_{n}(t)\right|^{2} W_{n}^{0}(x, k)$,

where again $\bar{N}$ is the cutoff mode number of the condensate band, given by (63).

\section{References}

[1] Pitaevskii L P 1961 Zh. Eksp. Teor. Fiz. 40646

[2] Gross E P 1961 Nuovo Cimento 20454

[3] Gross E P 1963 J. Math. Phys. 4195

[4] Dyachenko S, Newell A C, Pushkarev A and Zakharov V E 1992 Physica D 5796

[5] Semikoz D V and Tkachev I I 1995 Phys. Rev. Lett. 743093

[6] Semikoz D V and Tkachev I I 1997 Phys. Rev. A 55489

[7] Khlebnikov S and Tkachev I 2000 Phys. Rev. A 61083517

[8] Svistunov B V 1992 J. Mosc. Phys. Soc. 75387

[9] Kagan Y M, Svistunov B V and Shlyapnikov G V 1992 Sov. Phys._JETP 75387

[10] Gardiner C W and Zoller P 1997 Phys. Rev. A 552902

[11] Jaksch D, Gardiner C W and Zoller P 1997 Phys. Rev. A 56575

[12] Gardiner C W, Zoller P, Ballagh R J and Davis M J 1997 Phys. Rev. Lett. 791793

[13] Gardiner C W and Zoller P 1998 Phys. Rev. A 58536

[14] Jaksch D, Gardiner C W, Gheri K M and Zoller P 1998 Phys. Rev. A 581450

[15] Gardiner C W et al 1998 Phys. Rev. Lett. 815266

[16] Gardiner C W and Zoller P 2000 Phys. Rev. A 61033601

[17] Lee M D and Gardiner C W 2000 Phys. Rev. A 62033606

[18] Davis M J, Gardiner C W and Ballagh R J 2000 Phys. Rev. A 62063608

[19] Davis M J and Gardiner C W 2002 J. Phys. B: At. Mol. Opt. Phys. 35733

[20] Köhl M et al 2002 Phys. Rev. Lett. 88080402

[21] Davis M J, Ballagh R J and Burnett K 2001 J. Phys. B: At. Mol. Opt. Phys. 344487

[22] Drummond P D and Gardiner C W 1980 J. Phys. A: Math. Gen. 132353

[23] Steel M J et al 1998 Phys. Rev. A 584824

[24] Drummond P D and Corney J F 1999 Phys. Rev. A 60 R2661

[25] Deuar P and Drummond P D 2002 Phys. Rev. A 66033812

[26] Kagan Y and Svistunov B V 1997 Phys. Rev. Lett. 793331

[27] Marshall R J, New G H C, Burnett K and Choi S 1999 Phys. Rev. A 592085

[28] Stoof H T C 1999 J. Low Temp. Phys. 11411 
[29] Stoof H T C and Bilsma M J 2001 J. Low Temp. Phys. 124431

[30] Góral K, Gajda M and Rzążewski K 2001 Phys. Rev. Lett. 861397

[31] Góral K, Gajda M and Rzążewski K 2002 Phys. Rev. A 66 R051602

[32] Schmidt H et al 2003 J. Phys. B: At. Mol. Opt. Phys. 5 S96

[33] Zawitkowski L, Brewczyk M, Gajda M and Rzążewski K 2004 Phys. Rev. A 70033614

[34] Brewczyk M, Borowski P, Gajda M and Rzążewski K 2004 J. Phys. B: At. Mol. Opt. Phys. 372725

[35] Davis M J, Morgan S A and Burnett K 2001 Phys. Rev. Lett. 87160402

[36] Davis M J, Morgan S A and Burnett K 2002 Phys. Rev. A 66053618

[37] Davis M J and Morgan S A 2003 Phys. Rev. A 68053615

[38] Gardiner C W, Anglin J R and Fudge T I A 2002 J. Phys. B: At. Mol. Opt. Phys. 351555

[39] Gardiner C W and Davis M J 2003 J. Phys. B: At. Mol. Opt. Phys. 364731

[40] Sinatra A, Castin Y and Lobo C 2000 J. Mod. Opt. 472629

[41] Sinatra A, Lobo C and Castin Y 2001 Phys. Rev. Lett. 87210404

[42] Sinatra A, Lobo C and Castin Y 2002 J. Phys. B: At. Mol. Opt. Phys. 353599

[43] Lobo C, Sinatra A and Castin Y 2003 Phys. Rev. Lett. 92020403

[44] Lamb W E 1964 Phys. Rev. 134 A1429

[45] Tsubota M, Kasamatsu K and Ueda M 2002 Phys. Rev. A 65023603

[46] Choi S, Morgan S A and Burnett K 1998 Phys. Rev. A 574057

[47] Caradoc-Davies B M 1999 PhD Thesis University of Otago, Dunedin, New Zealand

[48] Dalfovo F, Giorgini S, Pitaevskii L P and Stringari S 1999 Rev. Mod. Phys. 71463

[49] Anglin J R and Zurek W H 1999 Phys. Rev. Lett. 831707

[50] Munro W J and Gardiner C W 1996 Phys. Rev. A 532633

[51] Gardiner C W and Zoller P 2004 Quantum Noise 3rd edn (Berlin: Springer)

[52] Penckwitt A A, Ballagh R J and Gardiner C W 2002 Phys. Rev. Lett. 89260402

[53] Cohen-Tannoudji C, Diu B and Lalöe F 1977 Quantum Mechanics vol 1 (New York: Wiley-Interscience)

[54] Penrose O and Onsager L 1956 Phys. Rev. 104576

[55] Blakie P B and Davis M J 2004 Preprint cond-mat/0410496

[56] Arfken G B and Weber H J 1995 Mathematical Methods for Physicists 5th edn (London: Academic) 